\title{
Risk factors of elderly patients with postoperative delirium following major abdominal surgery for
}

\section{cancer}

\author{
Seung Chul Heo, Hye Seong Ahn, Rumi Shin, Chang-Sup Lim, Dong-Seok Han \\ Department of Surgery, Seoul Metropolitan Government Seoul National University Boramae Medical Center, Seoul, Korea
}

Purpose: Postoperative delirium (POD) is a common complication in elderly patients after major abdominal surgery for cancer. Although POD is related with a poor outcome, there have not been many reports about POD after abdominal surgery in Korea. The aims of study were to analyze the characteristics and surgical outcomes of elderly patients with POD and to identify the risk factors of POD.

Methods: From November 2016 to January 2019, we prospectively enrolled 63 patients who were aged $\geq 75$ years and underwent major abdominal surgery for cancer. POD was daily assessed for up to 10 days postoperatively with the Confusion Assessment Method and a validated chart review.

Results: POD occurred in eight patients (12.7\%). Univariate analysis showed that the occurrence of POD was related to sodium $<135 \mathrm{mEq} / \mathrm{L}$ $(P=0.037)$, combined resection $(P=0.023)$, longer surgery/anesthesia time $(P=0.023$ and $P=0.037$, respectively), increased blood loss $(P=0.004)$, postoperative admission to intensive care unit $(I C U)(P=0.023)$, and duration of Foley catheter $(P=0.011)$, however, multivariate analysis identified no significant risk factors of POD. There was no difference in postoperative outcomes such as hospital stay, mortality, reoperation, and morbidity between patients with POD and without POD.

Conclusion: Elderly patients with hyponatremia, combined resection, longer operation/anesthesia time and admission to ICU had tendencies to develop POD after major abdominal surgery. Surgeons should pay more attention to prevent POD, and a large-scale prospective study is needed to identify the risk factors of POD.

Keywords: Delirium, Carcinoma, Risk factors, Elderly patients, Abdominal surgery

\section{INTRODUCTION}

The number of elderly patients undergoing major abdominal surgery is increasing. This trend will continue as life expectancy has

Received: Oct 15, 2020 Revised: Nov 2, 2019 Accepted: Nov 6, 2020 Correspondence to: Hye Seong Ahn

Department of Surgery, Seoul Metropolitan Government Seoul National University Boramae Medical Center, 20 Boramae-ro 5-gil, Dongjak-gu, Seoul 07061, Korea

Tel: +82-2-870-2276, Fax: +82-2-831-2826

E-mail: comette@snu.ac.kr

ORCID: Seung Chul Heo (https://orcid.org/0000-0003-3196-5158), Hye Seong Ahn (https://orcid.org/0000-0001-6853-7793), Rumi Shin (https://orcid.org/00000001-5500-8702), Chang-Sup Lim (https://orcid.org/0000-0002-2349-9647), Dong-Seok Han (https://orcid.org/0000-0002-9003-7987)

Copyright (c) 2020 Korean Society of Surgical Oncology

This is an Open Access article distributed under the terms of the Creative Commons Attribution Non-Commercial License (http://creativecommons.org/licenses/by-nc/4.0) which permits unrestricted non-commercial use, distribution, and reproduction in any medium, provided the original work is properly cited. improved and the population is aging. Understanding the risks for adverse outcomes in the aging surgical population is essential to increase safety and reduce costs. In elderly patients, delirium is known as the most common surgical complication with an incidence of $15 \%$ to $25 \%$ after major elective surgery and 50\% after high-risk procedures such as hip-fracture repair and cardiac surgery [1]. Furthermore, a strong correlation has been observed between increasing age and incidence of postoperative delirium (POD) after major surgery [2]. The official diagnosis of delirium by Diagnostic and Statistical Manual of Mental Disorders (DSM5) required following criteria: (1) disturbance in attention (i.e., reduced ability to direct, focus, sustain, and shift attention) and awareness (reduced orientation to the environment); (2) the disturbance develops over a short period of time (usually hours to a few days), represents an acute change from baseline attention and awareness, and tends to fluctuate in severity during the course of a day; (3) an additional disturbance in cognition; (4) the disturbances in criteria (1) and (3) are not better explained by a pre-existing, 
established, or evolving neurocognitive disorder and do not occur in the context of a severely reduced level of arousal such as coma; and (5) there is evidence from the history, physical examination or laboratory findings that the disturbance is a direct physiological consequence of another medical condition, substance intoxication or withdrawal, or exposure to a toxin, or is due to multiple etiologies [3]. POD is a potential risk factor for extended lengths of stay, higher patient care costs, morbidity, discharge to a post-acute nursing facility, and greater risk of death [4-6]. In Korea, there have been several reports on POD in patients after abdominal surgery, but most of them demonstrated POD in patients after liver transplantation [7-9]. The incidence of POD in elderly patients was reported as around $18.3 \%$ after major abdominal surgery [10], 8.6\%-41.2\% after gastrectomy [11,12], and 8.8\% after colorectal surgery [12]. These differences in incidence were related with the methods for diagnosis of POD. Many studies in Korea were retrospective or observatory studies using chart review or big data, which had tendencies to underestimate the incidence of POD.

This prospective study aimed to identify the incidence of POD after major abdominal surgery for cancer and to analyze the risk factors and surgical outcomes of elderly patients with POD.

\section{METHODS}

\section{Patients}

From November 2016 to January 2019, we prospectively enrolled elderly patients who were aged 75 years and greater and underwent major abdominal surgery. Patients with delirium at admission, emergent surgery, severe visual disturbance, dementia, and aphasia were excluded. Patients who were discharged within 5 days were also excluded. This study was approved by the Institutional Review Board of Seoul Metropolitan Government Seoul National University Boramae Medical Center (IRB No. 16-2014-1023/091) and written informed consent was obtained.

\section{Anesthesis, surgery, and postoperative course}

General anesthesia was used in all patients. It was induced with intravenous (IV) administration of fentanyl, propofol, and rocuronium without premedication, and was maintained with sevoflurane. At the end of surgery, the residual neuromuscular blockade was reversed with IV injection of glycopyrrolate, pyridostigmine and/ or sugammadex.

Surgical procedures were classified into four categories to assess the influence of each surgical procedure on the development of POD: (1) gastric surgery including partial or total gastrectomy; (2) colorectal surgery including colectomy and proctectomy; (3) hepatic surgery; or (4) biliary-pancreatic surgery. Laparoscopic sur- gery was frequently used except in patients presenting with low cardiopulmonary function or intrabdominal severe adhesion.

After surgery, all patients used IV patient-controlled analgesia using fentanyl and Aloxi in $100 \mathrm{~mL}$ of saline and/or morphine. The following were determined by the surgeons in charge; (1) postoperative admission to intensive care unit (ICU); (2) need to restrain of patients extremities; (3) date of removal of the nasogastric tube and Foley catheter; (4) date of restoration of a diet; or (5) discharge if no surgical or systemic complications occurred.

\section{Assessment and treatment of preoperative mental status and POD}

The presence of delirium was daily assessed for up to 10 days postoperatively with the Confusion Assessment Method (CAM) [13, 14], which has four criteria (acute onset and fluctuating course, inattention, disorganized thinking, and altered level of consciousness) and a validated chart review. When the patients were postoperatively admitted to the ICU, CAM-ICU was used [15]. All patients were seen at least once a day by a surgeon. Treatment of POD was monitored by a psychologist and was based on treatment of the eventual cause, and the administration of quetiapine. Antipsychotics medication such as haloperidol was avoided as much as possible since it could aggravate the severity and the duration of POD. Antipsychotic medication was only used to decrease the considerable agitation and aggressiveness observed in some patients with POD, who became dangerous to themselves and other caregivers.

\section{Data collection}

We collected preoperative data, anesthetic and surgical data and postoperative data.

Preoperative data included demography (gender, age, weight, height, education level, marital status, and heavy drinker) comorbidity, visual disturbance, the American Society of Anesthesiologists (ASA) classification, Eastern Cooperative Oncology Group performance status, preoperative cognitive function, activities of daily living using Barthel index, nutritional status, and laboratory test results. Cognitive function of the patients was assessed using the Mini-Mental State Examination-Korean (MMSE, score range of $0-30$ ) the day before surgery and all tests were conducted independently by a trained research nurse. Nutritional status was performed using Mini-Nutritional Assessment (MNA), which is well known to be an accurate nutritional assessment tool and is highly correlated with both clinical assessments of nutritional status and objective indicators such as serum albumin [16]. Nutritional status was divided to normal (24-30), risk of malnutrition (17-23.5), and malnutrition. Laboratory investigations included serum hemoglo- 
bin levels, serum electrolyte levels, the serum albumin level, and the serum calcium level.

Anesthetic and surgical data included type of surgical procedure, operative time, anesthetic time, intraoperative hypotensive event, estimated blood loss, and intraoperative transfusion.

Postoperative data included duration of Foley catheter, restrain, postoperative ICU stay, hospital stay, morbidity, mortality and readmission. Postoperative morbidity was graded based on the Clavien-Dindo classification [17], and according to this grading, grade III, IV, and V complications were classified as major complications.

\section{Statistics}

Patients' data were summarized using mean \pm standard deviation for continuous variables and frequency (\%) for categorical variables. Continuous variables were compared using the two-sample t-test and/or Wilcoxon rank-sum test. Categorical variables were compared using Pearson chi-square test or the Fisher exact test. A Cox proportional hazard model analysis was performed to identify risk factors for the development of POD.

A two-sided P-value of $<0.05$ was considered statistically significant. Statistical analysis was performed using IBM SPSS version 23 (IBM Corp., Armonk, NY, USA).

\section{RESULTS}

Among 143 eligible patients, only 67 patients agreed to participate in this study and four patients were excluded (two withdrawal of consent and two aborted surgery after discovering peritoneal seeding). Finally, 63 elderly patients were included.

Patients' preoperative characteristics were summarized in the Table 1. Mean age of patients was 79 years (range, $75-88$ years). Body mass index of 10 patients (15.9\%) was $20 \mathrm{~kg} / \mathrm{m}^{2}$ and less, and the ASA classification of all patients was 2. The score MMSE of 29 patients (46\%) was lower than 24.

POD was confirmed in eight patients (12.7\%), of which five patients $(62.5 \%)$ required antipsychotic medication. Seven patients (87.5\%) were diagnosed with POD, which developed within 4 days after surgery and occurred during the first postoperative week.

According to univariate analysis, the occurrence of $\mathrm{POD}$ was related to sodium $<135 \mathrm{mEq} / \mathrm{L}(\mathrm{P}=0.037)$, combined resection $(\mathrm{P}=0.023)$, duration of surgery $(\mathrm{P}=0.023)$, duration of anesthesia $(\mathrm{P}=0.037)$, estimated blood loss $(\mathrm{P}=0.004)$, postoperative admission to ICU ( $\mathrm{P}=0.023)$, and duration of Foley catheter $(\mathrm{P}=0.011)$ (Table 2). Multivariate analysis identified no significant risk factors of POD (Table 3).

Postoperatively, there was no reoperation and only one mortality in patients without POD. Eight patients with POD had similar
Table 1. Preoperative characters of elderly patients

\begin{tabular}{|c|c|}
\hline Variable & Value \\
\hline Age (yr) & $79.0 \pm 3.2$ \\
\hline Male sex & $43(68.3)$ \\
\hline $\mathrm{BMI}\left(\mathrm{kg} / \mathrm{m}^{2}\right)$ & $23.4 \pm 3.2$ \\
\hline Taking medication & $57(90.5)$ \\
\hline Visual disturbance & $3(4.8)$ \\
\hline \multicolumn{2}{|l|}{ ECOG } \\
\hline 0 & 48 (76.2) \\
\hline 1 & $13(20.6)$ \\
\hline 2 & $2(3.2)$ \\
\hline \multicolumn{2}{|l|}{ Education level } \\
\hline Elementary & $30(47.6)$ \\
\hline Middle school & $10(15.9)$ \\
\hline High school\&college & 19 (30.2) \\
\hline Alcohol drinker & $16(25.4)$ \\
\hline \multicolumn{2}{|l|}{ Preoperative blood tests } \\
\hline Hemoglobin $<11 \mathrm{~g} / \mathrm{dL}$ & $16(25.4)$ \\
\hline Creatinine $>1.4 \mathrm{~g} / \mathrm{dL}$ & $2(3.2)$ \\
\hline Total bilirubin $>1.2 \mathrm{mg} / \mathrm{dL}$ & $7(11.1)$ \\
\hline Albumin $<3.0 \mathrm{~g} / \mathrm{L}$ & $3(4.8)$ \\
\hline Sodium $<135 \mathrm{mEq} / \mathrm{L}$ & $12(19.0)$ \\
\hline Calcium $<8.6 \mathrm{mg} / \mathrm{dL}$ & $21(33.3)$ \\
\hline Preoperative MMSE & $24.2 \pm 3.2$ \\
\hline Preoperative score of activities of daily living & $19.8 \pm 1.2$ \\
\hline \multicolumn{2}{|l|}{ Preoperative nutritional status } \\
\hline Normal & $32(50.8)$ \\
\hline In risk & $21(33.3)$ \\
\hline Malnutrition & $9(14.3)$ \\
\hline \multicolumn{2}{|l|}{ Type of surgery } \\
\hline Gastric & $30(47.6)$ \\
\hline Colorectal & $25(39.7)$ \\
\hline Biliary-pancreatic & $6(9.5)$ \\
\hline Hepatic & $2(3.2)$ \\
\hline Laparoscopic approach & $45(71.4)$ \\
\hline Combined resection & $6(9.5)$ \\
\hline
\end{tabular}

Values are presented as mean \pm standard deviation or number (\%). BMI, body mass index; ECOG, Eastern Cooperative Oncology Group; MMSE, Mini-Mental State Examination-Korean.

hospital stay, 90-day mortality, reoperation and postoperative morbidity compared with 55 patients without POD (Table 4). The postoperative mean time to passage of flatus was $5.6 \pm 2.7$ days in eight patients with $\mathrm{POD}$ and $4.7 \pm 2.6$ days in 55 patients without POD ( $\mathrm{P}=0.450)$. The postoperative mean time to being able to tolerate a solid diet was $5.1 \pm 2.3$ in eight patients with POD and 4.9 \pm 1.8 in 55 patients without $\mathrm{POD}(\mathrm{P}=0.757)$. 
Table 2. Risk factors for postoperative delirium in 63 elderly patients

\begin{tabular}{|c|c|c|c|}
\hline Variable & POD $(n=8)$ & No POD $(n=55)$ & P-value \\
\hline Age (yr) & $80.6 \pm 3.2$ & $78.8 \pm 3.2$ & 0.123 \\
\hline Sex, M/F & $7 / 1$ & $36 / 19$ & 0.263 \\
\hline $\mathrm{BMI}\left(\mathrm{kg} / \mathrm{m}^{2}\right)$ & $21.8 \pm 2.4$ & $23.7 \pm 3.5$ & 0.079 \\
\hline Taking medicine & $8(100)$ & 49 (89.1) & 0.590 \\
\hline Comorbidity & $8(100)$ & $52(94.5)$ & 1.000 \\
\hline Visual disturbance & $1(12.5)$ & $2(3.6)$ & 0.339 \\
\hline Diabetes & $3(37.5)$ & $15(27.3)$ & 0.678 \\
\hline Cerebrovascular disease & $1(12.5)$ & $7(12.7)$ & 1.000 \\
\hline Cardiac disease except hypertension & $1(12.5)$ & $1(1.8)$ & 0.240 \\
\hline$E C O G, 0 / 1 \& 2$ & $6 / 2$ & $42 / 13$ & 1.000 \\
\hline Marital status (with spouse) & $8(100)$ & $52(96.3)$ & 0.580 \\
\hline Education level, middle school/high school\&college & $6 / 2$ & $34 / 17$ & 0.712 \\
\hline Alcohol drinker & $3(37.5)$ & $13(23.6)$ & 0.400 \\
\hline \multicolumn{4}{|l|}{ Preoperative blood tests } \\
\hline Hemoglobin $<11 \mathrm{~g} / \mathrm{dL}$ & $1(12.5)$ & $15(27.3)$ & 0.446 \\
\hline Creatinine $>1.4 \mathrm{~g} / \mathrm{dL}$ & 0 & $2(3.6)$ & 1.000 \\
\hline Total bilirubin $>1.2 \mathrm{mg} / \mathrm{dL}$ & $2(25.0)$ & $5(9.1)$ & 0.214 \\
\hline Albumin $<3.0 \mathrm{~g} / \mathrm{dL}$ & 0 & $3(5.8)$ & 1.000 \\
\hline Sodium < $135 \mathrm{mEq} / \mathrm{L}$ & $4(50.0)$ & $8(14.5)$ & 0.037 \\
\hline Calcium $<8.6 \mathrm{mg} / \mathrm{dL}$ & $5(62.5)$ & $16(29.1)$ & 0.104 \\
\hline Preoperative MMSE < 24 & $6(75.0)$ & $23(41.8)$ & 0.129 \\
\hline Preoperative score of activities of daily living & $19.9 \pm 0.4$ & $19.8 \pm 1.3$ & 0.594 \\
\hline Preoperative nutritional status, normal/in risk/malnutrition & $3 / 3 / 2$ & 29/18/7 & 0.680 \\
\hline Type of surgery & & & 0.491 \\
\hline Gastric & $3(37.5)$ & $27(49.1)$ & \\
\hline Colorectal & $3(37.5)$ & $22(40.0)$ & \\
\hline Biliary-pancreatic & $1(12.5)$ & $5(9.1)$ & \\
\hline Hepatic & $1(12.5)$ & $1(1.8)$ & \\
\hline Laparoscopic approach & $4(50.0)$ & $41(74.5)$ & 0.151 \\
\hline Combined resection & $3(37.5)$ & $3(5.5)$ & 0.023 \\
\hline Duration of surgery (min) & $242.5 \pm 71.4$ & $169.3 \pm 43.5$ & 0.023 \\
\hline Duration of anesthesia (min) & $282.8 \pm 73.8$ & $215.5 \pm 56.3$ & 0.037 \\
\hline Intraoperative hypotensive event & $3(37.5)$ & $11(20.0)$ & 0.361 \\
\hline Estimated blood loss (mL) & $518.8 \pm 411.4$ & $239.6 \pm 211.2$ & 0.004 \\
\hline Intraoperative transfusion & $2(25.0)$ & $2(3.2)$ & 0.077 \\
\hline Postoperative admission to ICU & $3(37.5)$ & $3(5.5)$ & 0.023 \\
\hline Duration of Foley catheter & $4.8 \pm 2.6$ & $3.3 \pm 1.3$ & 0.011 \\
\hline Restore sips of water (postoperative day) & $3.9 \pm 2.4$ & $3.5 \pm 1.5$ & 0.488 \\
\hline Postoperative morbidity & $1(12.5)$ & $4(7.3)$ & 1.000 \\
\hline
\end{tabular}

Values are presented as mean \pm standard deviation or number $(\%)$.

POD, postoperative delirium; M, male; F, female; BMI, body mass index; ECOG, Eastern Cooperative Oncology Group; MMSE, Mini-Mental State Examination-Korean; ICU, intensive care unit.

\section{DISCUSSION}

The incidence of POD was reported as an average $36.8 \%$ of surgical patients and varied from $9 \%$ to $87 \%$ depending on age, setting, type of surgery, other risk factors and the variation in screening practices $[4,18]$. Risk factors for POD have been categorized into predisposing and precipitating factors [4]. Older age, dementia (often not recognized clinically), functional disabilities, and a high burden of coexisting conditions are common predisposing factors. Male, poor vision and hearing, depressive symptoms, mild cogni- 
Table 3. Multivariate analysis of risk factors for postoperative delirium in 63 patients

\begin{tabular}{lcccc}
\hline Factor & P-value & Hazard ratio & \multicolumn{2}{c}{ 95\% Confidence interval } \\
\cline { 3 - 4 } & & & Lower & Upper \\
\hline Sodium $<135 \mathrm{mEq} / \mathrm{L}$ & 0.219 & 4.456 & 0.411 & 48.266 \\
Combined resection & 0.226 & 0.075 & 0.001 & 4.964 \\
Duration of surgery $(\mathrm{min})$ & 0.075 & 1.126 & 0.988 & 1.283 \\
Duration of anesthesia $(\mathrm{min})$ & 0.126 & 0.909 & 0.803 & 1.027 \\
Estimated blood loss $(\mathrm{mL})$ & 0.658 & 1.001 & 0.997 & 1.005 \\
Postoperative admission to ICU & 0.972 & 0.928 & 0.015 & 56.851 \\
Duration of Foley catheter & 0.338 & 1.352 & 0.730 & 2.504 \\
\hline
\end{tabular}

ICU, intensive care unit.

Table 4. Surgical outcomes of 63 elderly patients according to POD

\begin{tabular}{lccc}
\hline Factor & POD $(n=8)$ & No POD $(n=55)$ & P-value \\
\hline Hospital stay (day) & $14.1 \pm 6.1$ & $10.2 \pm 5.1$ & 0.142 \\
30-Day mortality & 0 & 0 & 1.000 \\
90-Day mortality & 0 & $1(1.8)$ & 1.000 \\
Reopeartion & 0 & 0 & 1.000 \\
Postoperative morbidity & $1(12.5)$ & $4(7.3)$ & 1.000 \\
Major morbidity & 0 & $1(1.8)$ & 1.000 \\
Wound infection & 0 & $2(3.6)$ & 1.000 \\
Pulmonary infection & $1(12.5)$ & $1(1.8)$ & 0.240 \\
Deep vein thrombosis & 0 & $1(1.8)$ & 1.000 \\
\hline
\end{tabular}

Values are presented as mean \pm standard deviation or number $(\%)$.

POD, postoperative delirium.

tive impairment, laboratory abnormalities, and alcohol abuse have also been known as predisposing factors [4]. On the other hand, drugs (especially sedative hypnotic agents and anticholinergic agents), surgery, anesthesia, high pain levels, anemia, infections, acute illness, and acute exacerbation of chronic illness are commonly reported precipitating factors [4]. Others reported that predictors of POD included delirium in medical history, advancing age per 10 years, ASA-score $\geq 3$, impaired mobility (timed get up and go test score $>20$ seconds) and postoperative tramadol administration [2,6]. According to Dhakharia et al. [19], respiratory complications, ICU stay $>24$ hours were also significantly associated with the POD.

This prospective study identified the incidence of POD as $12.6 \%$ in elderly patients aged 75 years and greater following major abdominal surgery for cancer in Korea. However, this study failed to identify the risk factors for POD and the association with poorer surgical outcomes in patients with POD. This failure might come from relatively low postoperatively morbidity and the small number of patients.

POD is usually known to negatively affect the postoperative course of clinical recovery, length of hospital stay, patient care costs, mor- bidity, discharge to a post-acute nursing facility, and greater risk of death [4-6]. Furthermore, major postoperative complications and delirium can demonstrate a strong combined effect and they are separately associated with adverse events [5]. However, that $30 \%$ to $40 \%$ of cases of delirium are preventable [20] led to trials finding the effective interventions to prevent POD. Those trials included orienting communication, oral and nutritional assistance, and early mobilization $[21,22]$. The patients were underwent the comprehensive geriatric assessment and got nutritional assistance to improve patients' preoperative health. Except these factors, surgeons have to do operation with good quality and short operative time to reduce the admission to ICU and postoperative pulmonary morbidity and surgeons should try not to use tramadol for the postoperative pain.

This study has several limitations. First, only $47 \%$ of eligible patients were included and this lowered the power of statistical analysis. The geriatric assessment included a large amount of inquiries and seemed burdensome. This could a hurdle in recruiting elderly patients. Second, the association between POD and the postoperative impairment of cognitive function could not be analyzed because the assessment of cognitive function was not postoperatively performed.

In summary, elderly patients with hyponatremia, combined resection, longer operation/anesthesia time, and admission to ICU had tendencies to develop POD after major abdominal surgery, although multivariate analysis failed to identify significant risk factors. Surgeons should pay more attention to prevent POD, and a large-scale prospective study is needed to identify the risk factors of POD.

\section{CONFLICT OF INTEREST}

No potential conflict of interest relevant to this article was reported. 


\section{ACKNOWLEDGMENTS}

This research was supported by Research Program 2015 funded by Seoul National University College of Medicine Research Foundation (800-20150342).

\section{REFERENCES}

1. Marcantonio ER. Postoperative delirium: a 76-year-old woman with delirium following surgery. JAMA 2012;308:73-81.

2. Brouquet A, Cudennec T, Benoist S, Moulias S, Beauchet A, Penna C, et al. Impaired mobility, ASA status and administration of tramadol are risk factors for postoperative delirium in patients aged 75 years or more after major abdominal surgery. Ann Surg 2010; 251:759-65.

3. European Delirium Association; American Delirium Society. The DSM-5 criteria, level of arousal and delirium diagnosis: inclusiveness is safer. BMC Med 2014;12:141.

4. Marcantonio ER. Delirium in hospitalized older adults. N Engl J Med 2017;377:1456-66.

5. Gleason LJ, Schmitt EM, Kosar CM, Tabloski P, Saczynski JS, Robinson T, et al. Effect of delirium and other major complications on outcomes after elective surgery in older adults. JAMA Surg 2015;150:1134-40.

6. Raats JW, van Eijsden WA, Crolla RM, Steyerberg EW, van der Laan L. Risk factors and outcomes for postoperative delirium after major surgery in elderly patients. PLoS One 2015;10:e0136071.

7. Yoon JS, Kim YR, Choi JW, Ko JS, Gwak MS, Kim GS. Risk factors of postoperative delirium following liver transplantation. Korean J Anesthesiol 2009;57:584-9.

8. Park KH, Son HJ, Choi YJ, Park GH, Lee YS, Park JY, et al. Liver transplant patients with high preoperative serum bilirubin levels are at increased risk of postoperative delirium: a retrospective study. J Clin Med 2020;9:1591.

9. Ri HS, Choi YJ, Park JY, Jin SJ, Lee YS, Son JM, et al. Elevation of preoperative ammonia level is not associated with the incidence of postoperative delirium in patients with liver transplantation: a propensity score matching analysis. Transplant Proc 2020;52:219-26.

10. Park EA, Kim MY. Postoperative delirium is associated with negative outcomes and long-term mortality in elderly Koreans: a retrospective observational study. Medicina (Kaunas) 2019;55:618.
11. Shin YH, Kim DK, Jeong HJ. Impact of surgical approach on postoperative delirium in elderly patients undergoing gastrectomy: laparoscopic versus open approaches. Korean J Anesthesiol 2015;68: 379-85.

12. Kang SY, Seo SW, Kim JY. Comprehensive risk factor evaluation of postoperative delirium following major surgery: clinical data warehouse analysis. Neurol Sci 2019;40:793-800.

13. Inouye SK, van Dyck CH, Alessi CA, Balkin S, Siegal AP, Horwitz RI. Clarifying confusion: the Confusion Assessment Method: a new method for detection of delirium. Ann Intern Med 1990;113: 941-8.

14. Wei LA, Fearing MA, Sternberg EJ, Inouye SK. The Confusion Assessment Method: a systematic review of current usage. J Am Geriatr Soc 2008;56:823-30.

15. Heo EY, Lee BJ, Hahm BJ, Song EH, Lee HA, Yoo CG, et al. Translation and validation of the Korean Confusion Assessment Method for the intensive care unit. BMC Psychiatry 2011;11:94.

16. Barone L, Milosavljevic M, Gazibarich B. Assessing the older person: is the MNA a more appropriate nutritional assessment tool than the SGA? J Nutr Health Aging 2003;7:13-7.

17. Dindo D, Demartines N, Clavien PA. Classification of surgical complications: a new proposal with evaluation in a cohort of 6336 patients and results of a survey. Ann Surg 2004;240:205-13.

18. McDaniel M, Brudney C. Postoperative delirium: etiology and management. Curr Opin Crit Care 2012;18:372-6.

19. Dhakharia V, Sinha S, Bhaumik J. Postoperative delirium in Indian patients following major abdominal surgery for cancer: risk factors and associations. Indian J Surg Oncol 2017;8:567-72.

20. Siddiqi N, House AO, Holmes JD. Occurrence and outcome of delirium in medical in-patients: a systematic literature review. Age Ageing 2006;35:350-64.

21. Chen CC, Li HC, Liang JT, Lai IR, Purnomo JDT, Yang YT, et al. Effect of a modified hospital elder life program on delirium and length of hospital stay in patients undergoing abdominal surgery: a cluster randomized clinical trial. JAMA Surg 2017;152:827-34.

22. Janssen TL, Mosk CA, van Hoof-de Lepper CC, Wielders D, Seerden TC, Steyerberg EW, et al. A multicomponent prehabilitation pathway to reduce the incidence of delirium in elderly patients in need of major abdominal surgery: study protocol for a before-and-after study. BMC Geriatr 2019;19:87. 